\title{
Pôster Quiz como Estratégia Inovadora na Exposição de Trabalhos em um Congresso de Educação Médica
}

\section{Poster-Quiz as an Innovative Strategy in the Presentation of Works at a Medical Training Congress}

\author{
Bráulio Brandão Rodrigues ${ }^{\circledR}$ \\ Lanna Tarce Gonçalves de Morais ${ }^{I}$ \\ Paola Souza Manzi ${ }^{I}$ \\ Isabella Françoise Teles ${ }^{I}$ \\ Kálita Oliveira Lisboa ${ }^{I}$ \\ Nathalia Vitória Ramos \\ Rafael Felipe Gregório Machado ${ }^{I}$ \\ Denis Masashi Sugital
}

\section{PALAVRAS-CHAVE}

- Educação Médica.

- Atividades Científicas e Tecnológicas.

- Comunicação e Divulgação Científica.

- Inovação.

\section{RESUMO}

Introdução: A exposição de trabalhos científicos é uma das metodologias utilizadas nos congressos e faz parte da comunicação informal do conhecimento científico, usada entre os cientistas ao se inserirem em um cotidiano restrito de pesquisadores. A tecnologia $Q R$ code facilita o acesso à produção científica e à dinamização do conhecimento; o Pôster Quiz constitui uma ferramenta de exploração dos trabalhos científicos facilitada pela inserção do QR code. Objetivos: Relatar a criação e elaboração do Pôster Quiz como estratégia inovadora na exposição de trabalhos em um congresso de educação médica, aliada a uma ferramenta digital, o QR code, a fim de incentivar a leitura dos trabalhos expostos nos totens digitais, disseminando a pesquisa científica, seus autores e sua importância. Relato de experiência: Trata-se de uma metodologia desenvolvida por discentes e docentes do curso de Medicina do Centro Universitário de Anápolis (UniEVANGÉLICA), em contou com reuniões periódicas que propunham a elaboração de $Q R$ codes que expusessem perguntas sobre resumos previamente lidos pelos participantes em formato e-poster. Esse processo envolveu a participação de sete discentes, um docente e 47 congressistas. Utilizaram-se banners com QR codes correspondentes às perguntas de seis totens de e-poster espalhados pelo evento, cartões-resposta referentes às perguntas expostas pelos QR codes e uma urna para depósito dos cartões; a correção dos cartões foi automática. Ao encontrarem um banner, os congressistas utilizavam um aplicativo leitor de $Q R$ code para obter acesso às questões daquele totem no período específico e em seguida tinham que realizar uma leitura dos trabalhos expostos ou assistir à apresentação do e-poster para que pudessem responder às questões. A correção dos cartões-resposta ocorreu por meio do aplicativo mobile Merrit $\AA_{\text {, }}$ que utiliza a câmera do smartphone para identificar as respostas de questões objetivas, comparando-as com um gabarito previamente informado. Do total de 150 questões, os primeiros colocados apresentaram 77 (51,33\%), 76 (50,66\%) e 70 (46,66\%) acertos, sendo que a média de acertos foi de 61 (40,66\%). Ao término do evento, ocorreu a premiação dos ganhadores nas três primeiras colocações. Também foram coletados relatos pessoais dos congressistas, atribuindo credibilidade a essa prática. Conclusão: A importância dessa dinâmica repousa, sobretudo, na valorização dos trabalhos expostos com o objetivo de estimular o interesse dos congressistas, além de incentivar a integração entre os participantes da dinâmica e despertar maior atenção para as informações prestadas. Vale ressaltar o impacto positivo de uma atividade distinta de todas as outras do congresso, que agregou um elemento competitivo favorável ao objetivo primordial de obter conhecimento. O uso dessa nova tecnologia teve um resultado auspicioso, visto que a criação de um desafio premiado promoveu benefícios intelectuais, como a aquisição de habilidades de leitura e o aprendizado de temas pouco retratados na faculdade. A eficácia dessa dinâmica reside numa troca proveitosa de conhecimentos e de interesse entre os participantes, os autores dos trabalhos e os elaboradores do Pôster Quiz. 


\section{KEY-WORDS}

- Medical Education.

- Scientific and Technical Activities.

- Scientific Communication and Dissemination.

- Innovation.

\begin{abstract}
Introduction: Scientific works are routinely presented at medical congresses, contributing to the informal communication of scientific knowledge among scientists in a context of a restricted number of researchers. $Q R$ codes facilitate access to scientific production and promote more dynamic learning, and the Poster-Quiz is a tool we used to explore such works by means of QR codes. Objectives: To report on the creation and development of a Poster-Quiz as an innovative form of displaying works at a medical congress, with a view to encouraging reading of the works exhibited in the digital totems, disseminating and highlighting the importance of scientific research and its authors. Experience report: A methodolody developed by students and professors of the Medicine course at UniEVANGÉLICA - University Centre of Anápolis, involving periodic meetings to propose the use of $Q R$ codes to set questions about previously read abstracts displayed in e-posters. This process included the participation of seven students, one professor and 47 congress participants. The experiment was conducted with the use of banners with $Q R$ codes corresponding to questions about the content of six digital totems spread around the event, response cards and an urn for collection of the cards, which were corrected automatically. Upon finding a banner, the congress participants used a $Q R$ code reader app to access the questions relative to that totem in the specific period and then had to read the works presented or watch a presentation of the e-poster in order to answer the questions. The mobile app Merrit $\AA$, using a smartphone camera was employed to identify the answers to the objective questions, comparing them to a previously established answer sheet. There were 150 quiz questions in all and the top-performing respondents achieved scores of $51.33 \%, 50.66 \%$ and $46.66 \%$ correct answers. The average score was around $40.66 \%$ correct answers. At the end of the event, the top three respondents were awarded. The participants were also asked to give their opinion on the quiz experience in order to lend credibility to the practice. Conclusion: The importance of this activity resides, above all, in promoting the works exposed in order to spark the interest of the congress participants, as well as encouraging greater integration between the participants and provoking them to pay closer attention to the information exhibited. We should highlight the positive impact of an activity which is clearly different to the others found at the congress, and which added a competitive element that enhanced the main objective of learning. The use of this new technology had an auspicious outcome, seeing as the creation of a competitive challenge promoted intellectual benefits, such as the aquisition of reading abilities and learning about matters that are given little attention at university. The effectiveness of the quiz derives from a mutually beneficial exchange of knowledge and interest among the participants, authors of the works and the organizers of the Poster-Quiz.
\end{abstract}

Recebido em: 20/3/19

Aceito em: 8/4/19

\section{INTRODUÇÃO}

Os congressos científicos são reuniões de profissionais interessados em atualização ou discussão de temas específicos de grande relevância para o contexto da temática e se destinam a um público seleto, com a utilização de diversas metodologias para expor o assunto-alvo. Esses eventos, em que a produção científica tem grande destaque ${ }^{1}$, facilitam o acesso a novos conhecimentos científicos pela comunidade acadêmica e divulgam este saber à humanidade, além de constituírem um canal de comunicação científica, promovendo também o reconhecimento a quem produz ${ }^{2}$.

A exposição de trabalhos científicos é uma das metodologias utilizadas nos congressos e faz parte da comunicação informal do conhecimento científico por instituir-se no cotidiano de um número restrito de pesquisadores em contraposição à comunicação formal, na qual se enquadram os livros e perió- dicos científicos. A motivação para expor o trabalho decorre da oportunidade de aperfeiçoá-lo por meio do conhecimento advindo do evento e, finalmente, formalizar suas contribuições para vetores formais ${ }^{3,4}$. Assim, atividades inovadoras nesse ambiente são de grande valor, pois, atualmente, nos cursos universitários médicos, o ensino requer um pensamento ousado, que não se limite às demandas de modelos antigos de aprendizagem, mas inclua a lucidez de obter uma formação competente nas situações factuais ${ }^{5}$.

No meio acadêmico, a apresentação de trabalhos científicos é o meio de comunicação informal eleito entre os cientistas, pois reúne informações recentes, seletas, concentradas e oportunas, e elenca pessoas que podem trabalhar a serviço da ciência ${ }^{2}$. Além disso, ter trabalhos apresentados em eventos científicos compõe o currículo paralelo do estudante, em que ele produz atividades extracurriculares de ensino, pesquisa e extensão a 
fim de pleitear vagas em certames e processos de pós-graduação ${ }^{6}$. Entre as modalidades de apresentação, há os pôsteres, impressos (lona, papel, etc.) ou virtuais, que utilizam a comunicação escrita e visual; e a comunicação oral, que emprega recursos audiovisuais para transmitir a divulgação científica. Por esses meios, os expositores são avaliados quanto a postura, conteúdo, expressões e utilização de elementos visuais ${ }^{7}$.

A educação médica tem sofrido mudanças evidentes em relação tanto à sua estruturação, quanto à inserção de tecnologias no ensino. Temos como exemplo o Quick Response (QR) Code, um instrumento de grande valia, que consiste numa leitura ótica em que códigos bidimensionais são decodificados por scanners e leitores compatíveis com a tecnologia ${ }^{8}$. O QR code foi criado no Japão em 1994 por uma empresa do grupo Toyota. Inicialmente destinado a peças automotivas, se disseminou para o mundo todo e atualmente é utilizado em várias funções ${ }^{9}$.

$\mathrm{O} Q R$ code faz transitar a informação, reduzindo trajetórias e potencializando o acesso à informação virtual ${ }^{10}$, funcionando, assim, como um hiperlink, fazendo com que atividades de educação médica se reduzam a um instante (ou um "clique"). Dessa forma, os recursos didáticos que podem ser gerados tornam o processo de aprendizagem mais fácil e instigante, tirando expositor e leitor da inércia e trazendo resultados positivos. Metodologias ativas nas universidades também usam $Q R$ codes, propiciando o protagonismo do estudante, a fixação do conteúdo e maior interação com a tecnologia desenvolvida para o ensino-aprendizagem ${ }^{11}$.

Assim como o ensino médico tem apostado no uso das tecnologias, os congressos acadêmicos também têm inovado, utilizando aplicativos em smartphones para que os congressistas acessem materiais e façam perguntas aos palestrantes ao final de suas exposições, proporcionando o anonimato e desenvolvendo o aprendizado. $\mathrm{O}$ uso das tecnologias em eventos médicos permite uma participação mais intensa e efetiva, aumentando a assimilação dos temas e o interesse em participar de estratégias semelhantes, tornando os eventos cada vez maiores $^{12}$.

As dinâmicas de valorização da produção cientifica com incentivos aos participantes e fazendo uso de tecnologia, além de contemplarem a proposta inovadora, ratificam a ideia de educação médica contemporânea ${ }^{13}$. Tais estratégias geram profissionais que não apenas são tecnicamente peritos em seu território de atuação e didática, mas também trabalham com novos linguajares e metodologias de obtenção do conhecimento ${ }^{14}$.

Este trabalho tem como objetivo relatar a criação e elaboração do Pôster Quiz como estratégia inovadora na exposição de trabalhos em um congresso de educação médica. Esta metodologia visa destacar a exposição dos trabalhos científicos no congresso (que, muitas vezes, fica por conta dos congressistas), aliada a uma ferramenta digital, $Q R$ code, a fim de incentivar a leitura dos trabalhos expostos nos totens digitais, divulgando a pesquisa científica, seus autores e sua importância.

\section{RELATO DE EXPERIÊNCIA}

Trata-se de um relato de experiência da metodologia Pôster Quiz, desenvolvida por discentes e docentes do curso de Medicina do Centro Universitário de Anápolis (UniEVANGÉLICA). A atividade foi desenvolvida de março a agosto de 2018, constituindo-se de vários encontros vivenciais na UniEVANGÉLICA. Essas reuniões visavam à elaboração de $Q R$ codes para que os congressistas pudessem ter acesso a questões sobre os trabalhos que seriam apresentados durante o VII Congresso de Educação Médica do Centro Oeste (Coemco), a partir da leitura dos resumos aprovados para apresentação em formato $e$-poster e a construção de perguntas e respostas para cada trabalho. Todo o processo envolveu a participação de sete discentes, do primeiro ao décimo período do curso de Medicina da UniEVANGÉLICA, orientados por um docente da instituição, que constituíram a Comissão Organizadora da metodologia e são os autores do presente trabalho.

Foi elaborado um edital sobre a metodologia do Pôster Quiz, que foi divulgado nas redes sociais do VII Coemco 30 dias antes da data do evento, para que todos os congressistas soubessem da dinâmica antes do evento. Entre os vários trabalhos submetidos para apresentação no VII Coemco, foram selecionados os 150 resumos que seriam apresentados em forma de $e$-poster no evento. A Comissão Organizadora distribuiu esses trabalhos entre seus membros para a elaboração de questões objetivas, de múltipla escolha, com base na leitura minuciosa dos resumos desses trabalhos. Elaborou-se uma pergunta para cada trabalho, cada uma com quatro opções de resposta. No total, o Pôster Quiz constituiu-se de 150 perguntas de nível de dificuldade variável, havendo perguntas que exigiam menos conhecimento do participante - abordando algo referente a metodologia ou eixo temático, por exemplo e outras que requeriam conhecimento prévio de metodologia científica ou uma leitura mais atenciosa do trabalho.

As perguntas foram elaboradas seguindo os cinco eixos temáticos do evento: currículo e metodologias de ensino; humanização, ética e profissionalismo; novas tecnologias aplicadas no ensino em saúde; saúde mental no ensino médico; gestão, formação de lideranças e compromisso social no ensino médico. $\mathrm{O}$ conteúdo cobrado nessas perguntas abordava diversos tópicos, como linha de pesquisa, eixo temático, objetivo do trabalho, metodologia, resultados e discussão, de modo a valorizar o processo científico utilizado pelos autores dos resumos. 
Cada questão foi revisada pelo docente orientador a fim de assegurar que as respostas exigissem a leitura e o entendimento do conteúdo do pôster. Esse procedimento mostrou-se importante para corrigir possíveis vieses de abordagem ou falta de clareza e objetividade na redação das perguntas, de maneira a evitar anulação de questões por ambiguidade, incoerência ou existência de mais de uma alternativa correta. Após o processo de revisão, foram criados $Q R$ codes, contendo, cada um, de duas a três perguntas da dinâmica. Esses $Q R$ codes foram disponibilizados ao lado dos totens que exibiam os trabalhos referentes às perguntas. Cada participante do Pôster Quiz teve acesso às questões por meio de aplicativo leitor de $Q R$ code instalado em seu smartphone. Os totens foram espalhados estrategicamente pelas áreas do evento a fim de garantir maior circulação dos participantes. Cada totem tinha, em média, de seis a nove trabalhos expostos por turno, sendo cinco totens espalhados durante os dois dias de congresso (Figura 1).

Durante o evento, os participantes da dinâmica tiveram que realizar uma breve inscrição para dar início ao Pôster Quiz. Após se cadastrarem, receberam um cartão-resposta para assinalar a alternativa que julgassem correta, preenchen-

\section{Figura 1}

Banner com o $Q R$ code com perguntas sobre os trabalhos de determinado totem em um turno do evento
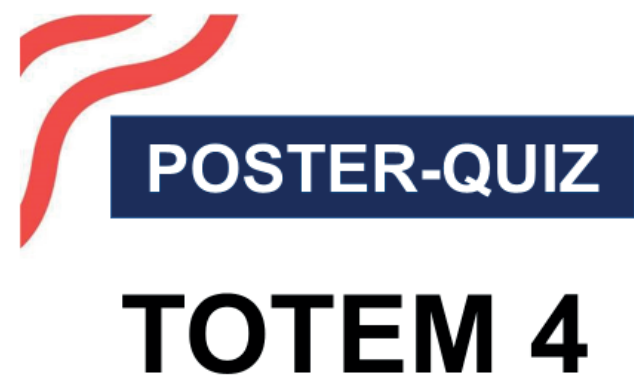

\section{Turno Matutino}

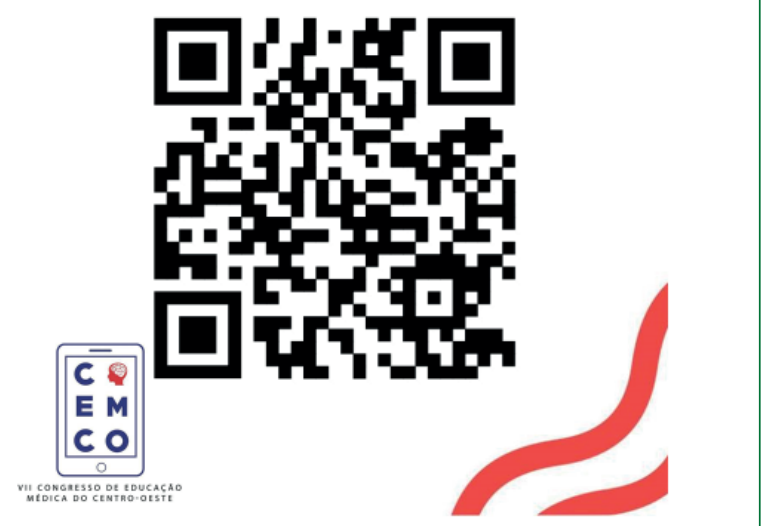

Fonte: Acervo pessoal dos autores. do o campo específico para cada questão (Figura 2). Os congressistas procuravam todos os banners durante o congresso. Ao encontrarem um banner, utilizavam um aplicativo leitor de $Q R$ code para obter acesso às questões daquele totem no período específico; em seguida, tinham que realizar uma leitura dos trabalhos expostos ou assistir à apresentação do e-poster para que pudessem responder às questões.

Figura 2

Cartão-resposta utilizado em um dos turnos do evento

\section{CONGRESSO DE EDUCAÇÃO MÉDICA DO CENTRO-OESTE}

Nome:

Telefone:

CARTÃO RESPOSTA

CURRICULO OCULTO E FORMAÇÃO MÉDICA: A IMPORTÂNCIA DA MONITORIA DE EXTENSÁO NO DESENVOLVIMENTO DE HABILIDADES
(A) (B) (C) (D) ÀS MARGENS DO RIO E DA
SOCIEDADE: CUIDANDO DA SAÚDE
DE COMUNIDADES RIBEIRINHAS DA AMAZÔNIA

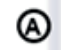

(B)

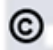

(D)

PITORESCO PODER DA CURA PELO CÓMICO: DESENVOLVENDO A PALHAÇOTERAP
HOSPITALAR

\section{(A)$$
\text { (B) }
$$

(C)

INTERAÇÕES ENTRE O APRENDER, ENSINAR E SOCIALIZAR: MONITORIA DE EXTENSÃO, UMA FORMA DE ARTICULAÇÄO ENTRE A VIDA ACADÉMCIA EA SOCIEDADE

\section{(A) \\ (B) \\ (C) \\ (2)}

ENSINO À DISTÂNCIA COMO FERRAMENTA PARA A EDUCÇÃO EM SAÚDE COM ENFASE NA MEDICINA BASEADA EM EVIDÉNCIAS: UM RELATO DE EXPERIÊNCIA
(A)
(B)
()
(2) P ACOLLIMENTO NA ATENÇÃo IMPORTANTE INSTRUMENTO DE VINCULO E RESOLUTVIDADE: UM RELATO DE EXPERIÊNCIA

(A) (B) (C)

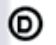

DIAGNÓSTICO SITUACIONAL DE UMA COMUNIDADE REALIZADO POR ESTUDANTES DE MEDICINA ATRAVÉS DA TÉCNICA DE ESTIMATIVA RÁPIDA PARTICIPATIVA

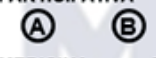

MEDICINA REPLICADA NO LABORATÓRIO: REPLICADA NO LABORATORIO: EMERGENCIAS CIRURGICAS ATRAVES DA SIMULAÇĀO AVANÇADA COM ACIENTES ATOR

(A)

(B)

(C) (2)

DISCUTINDO DIVERSIDADE SEXUAL NO MEIO ACADÉMICO: UM RELATO DE EXPERIÉNCIA

(A) B) (C) (D)

OBJETIVOS DE APRENDIZAGEM NO MODULO DOENCAAS RESULTANTES DE AGRESSÖES AO MEIO AMBIENTE: APLICANDO A TAXONOMIA REVISADA DE BLOOM

DIA 2 - TURNO MATUTINO UnIEVANGÉLICA

No final de cada turno, os participantes depositavam seus cartões-resposta numa urna (Figura 3) localizada em local estratégico, próximo ao midia-desk, de fácil acesso a todos. A fim de manter a imparcialidade, a Comissão Organizadora não participou da dinâmica, e os cartões-resposta foram recolhidos uma única vez a cada turno e colocados num envelope, para posterior correção. Participaram dessa metodologia 47 congressistas, discentes (em sua maioria) e docentes, com representação da maioria das instituições de ensino superior presentes no evento.

A correção dos cartões-resposta ocorreu por meio do aplicativo mobile Merrit ${ }^{\circ}$, que utiliza a câmera do smartphone para identificar as respostas de questões objetivas, comparando-as com um gabarito previamente informado. Essa correção foi 


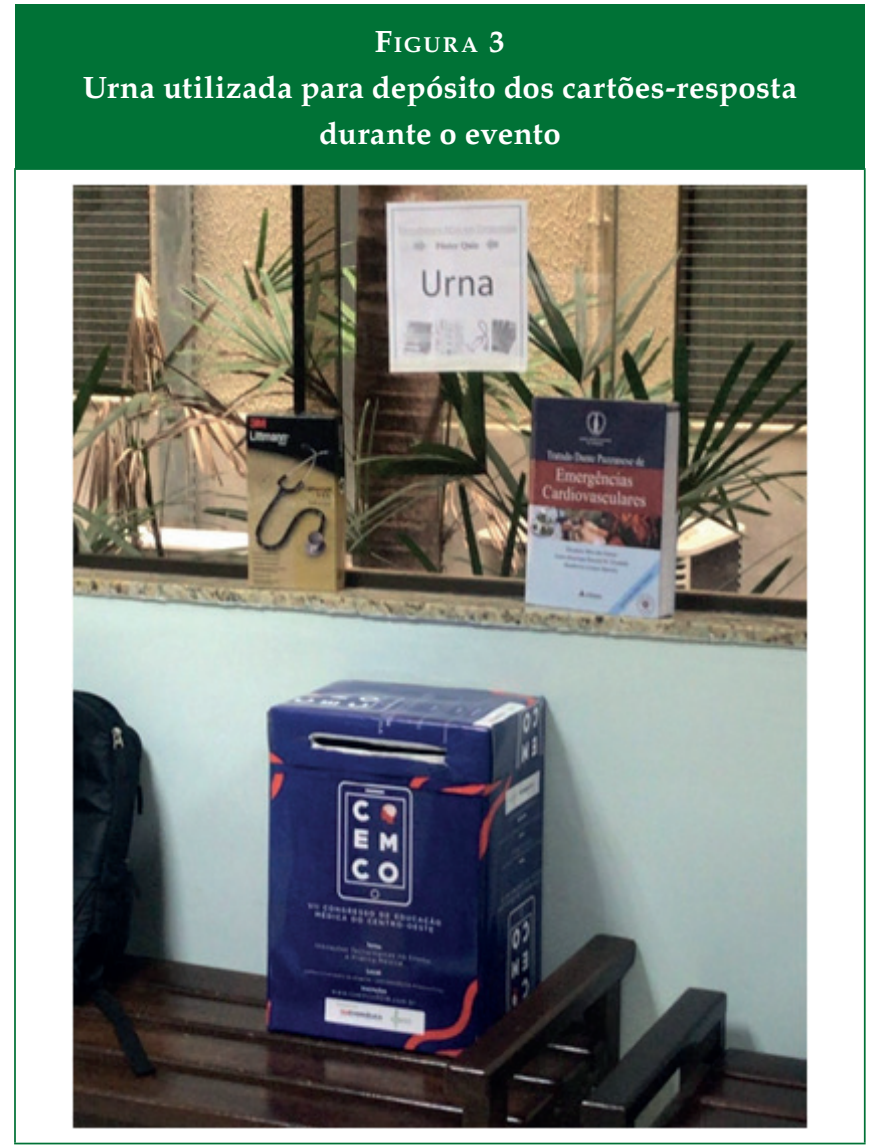

Fonte: Acervo pessoal dos autores.

realizada por dois aparelhos diferentes, a fim de minimizar possíveis interferências advindas do uso desta tecnologia. Do total de 150 questões, os primeiros colocados apresentaram 77 $(51,33 \%), 76(50,66 \%)$ e $70(46,66 \%)$ acertos, sendo que a média de acertos foi de $61(40,66 \%)$. Os participantes tenderam a acertar mais questões no primeiro dia do evento, com maior percentual de erros posteriormente. Ao término do evento, ocorreu a premiação dos ganhadores nas três primeiras colocações, sendo que três ganhadores empataram na terceira posição. Segundo um dos ganhadores, a premiação foi um fator que estimulou a participação na atividade:

Normalmente, os alunos não leem os trabalhos apresentados. Com um desafio premiado, os participantes do evento têm maior interesse em participar das apresentações de trabalhos. (ganhador do $3^{\circ}$ lugar)

Durante a premiação, os ganhadores foram entrevistados sobre as impressões e autoavaliação da atividade, vantagens em relação ao método tradicional de apresentações de trabalhos e dificuldades apresentadas no decorrer da atividade:
No geral, a experiência foi positiva, me incentivou a ler trabalhos e, consequentemente, aprendi a ler também artigos com mais rapidez e objetividade. Além disso, tive vários exemplos de bons resumos a serem enviados a congressos, o que foi muito bom pra mim, uma vez que comecei o curso há pouco tempo e ainda não sabia como fazê-los. O ponto negativo foi a quantidade de resumos para o pouco tempo que havia para responder às questões. Me dediquei muito a responder a um quiz e acabei não participando de tantas atividades do próprio congresso. (ganhador do $1^{\circ}$ lugar)

Me ajudou a aprender sobre novas doenças, novas pesquisas e novas associações, que eu provavelmente não iria ver na faculdade. (ganhador do $2^{\circ}$ lugar)

Os participantes, informalmente, relataram gostar da metodologia, alegando o caráter interativo promovido durante a apresentação de trabalhos, associado ao estímulo competitivo e às premiações para aqueles que mais se destacassem. Como pontos negativos, alegaram a enorme quantidade de trabalhos e o fato de estes não ficarem expostos continuamente nos totens. Alguns relataram que deixaram de participar de atividades do congresso para se dedicarem à dinâmica.

Como vantagens em relação ao método tradicional, esses participantes referiram um estímulo positivo à leitura dos trabalhos apresentados no evento, o que, na maioria das vezes, é uma atividade monótona e pouco interessante. Além disso, ressaltaram o desenvolvimento da habilidade de leitura dinâmica e acesso a metodologias de trabalhos e temas nem sempre disponíveis no cotidiano universitário.

\section{CONCLUSÃO}

A importância da dinâmica utilizada repousa, sobretudo, na valorização dos trabalhos expostos, visando estimular o interesse dos congressistas pelos diferentes temas abordados por meio de uma atividade lúdica e inovadora. Além disso, a integração entre os participantes do Pôster Quiz e o despertar de maior atenção para as informações contidas nos pôsteres contribuem para um melhor aprendizado e aproveitamento do evento. Apesar da dificuldade de se adaptar a uma nova metodologia de aprendizagem, o prospecto para eventos futuros e a contribuição acadêmica de forma inovadora se sobrepuseram a quaisquer contratempos ou obstáculos de adesão. Vale ressaltar o impacto positivo de uma atividade distinta de todas as outras do congresso, que agregou um elemento competitivo positivo ao objetivo primordial de obter conhecimento.

Nota-se a eficácia desse novo elemento de ensino e de agregação por meio do interesse dos congressistas em conhe- 
cer mais sobre o proposto, tendo como ponto de partida a importância de atentar para a metodologia e o conteúdo dos trabalhos. Dessa forma, houve um incremento da participação no congresso, seja pelo aprendizado de temas extracurriculares do ambiente acadêmico, seja pelo desenvolvimento de habilidades de cognição. Prova disso são os relatos dos participantes da atividade, que ressaltaram que o estímulo à competitividade trouxe inovações que permanecerão além do congresso. Segundo um dos ganhadores: "[...] além de bons exemplos de resumos, mais conhecimento, adquiri habilidades maiores em fazer leituras mais dinâmicas ao pesquisar trabalhos/artigos".

Outro ponto relevante exposto foi o de que a existência de perguntas forçava os congressistas a ler criteriosamente os trabalhos e, até mesmo, buscar informações adicionais acerca dos assuntos. Isso despertou neles uma visão diferente das apresentações, frequentemente tidas como monótonas e desinteressantes.

Conclui-se, portanto, que o uso dessa nova tecnologia teve um resultado positivo para o congresso, visto que a criação de um desafio premiado promoveu benefícios intelectuais, como a aquisição de habilidades de leitura e o aprendizado de temas pouco retratados na faculdade. Assim, a eficácia dessa dinâmica reside numa troca proveitosa de conhecimentos e de interesse entre os participantes, os autores dos trabalhos e os elaboradores do Pôster Quiz.

\section{REFERÊNCIAS}

1. Andrade RB. Manual de Eventos. $2^{\underline{a}}$ ed. ampl. Caxias do Sul: EDUCS, 2002.

2. Guimarães VAL, Innocentini Hayashi, MCP. Os Eventos Científicos: espaços privilegiados para a comunicação da ciência. Comunicologia - Revista de Comunicação da Universidade Católica de Brasília 2015, 7(2), 204-229.

3. Moreno F, Arellano MAM. Publicação científica em arquivos de acesso aberto. Arquivistica. Net2005, 1(1).

4. Mueller SPM. A publicação da ciência: áreas científicas e seus canais preferenciais. Datagrama Zero: Revista de Ciência da Informação 2005, 6 (1), 1-12.

5. Batista SHSS. A interdisciplinaridade no ensino médico. Revista Brasileira de Educação Médica 2006, 30 (1), 39-46.

6. Tavares AP et al. O "Currículo Paralelo" dos estudantes de medicina da Universidade Federal de Minas Gerais. Revista Brasileira de educação médica 2007, 31 (3), 254-265.

7. Carmo JS, Prado PST. Apresentação de trabalho em eventos científicos: comunicação oral e painéis. Interação em Psicologia 2005, 9 (1), 131-142.

8. Parra F. Mídias digitais: reapropriação da tecnologia QR Code. Anais da III Conferência do Pensamento Comu- nicacional Brasileiro (PREZACOM) [on-line]. 2016, 1 (1) [capturado em 12 dez. 2018]. Disponível em: http:/ / www. portalintercom.org.br/anais/pensacom2016/textos/felipe-parra.pdf

9. Soon TJ. QR code. Synthesis Journal 2008, 1 (1),59-78.

10. Parra F. Reflexões sobre as relações entre usuário-interator e tecnologias emergentes a partir do QR Code. Temática 2018, 14(5), 156-172.

11. Nicola JA, Paniz CM. A importância da utilização de diferentes recursos didáticos no Ensino de Ciências e Biologia. InFor 2017, 2 (1), 355-381.

12. Saldanha M. Congresso Médico de Maringá discute o uso da tecnologia na medicina. [on-line]. 2018. [capturado 01 dez. 2018]. Disponível em: https://maringapost.com.br/ cidade/2018/10/09/congresso-medico-de-maringa-discute-o-uso-da-tecnologia-na-medicina-serao-mais-de-30-palestras-entre-11-e-13-de-outubro-sobre-impressao-de-orgaos-realidade-virtual-e-outros-temas /

13. Garcia W. Entre Comunicação, Consumo e Tecnologia: Apontamentos sobre a Blogosfera. Anais da III Conferência do Pensamento Comunicacional Brasileiro (PREZACOM) [on-line]. 2016, 1 (1) [capturado em 30 dez. 2018]. Disponível em: http://www.portalintercom.org.br/anais/pensacom2016/textos/wilton-garcia.pdf

14. Abensur SI, Tamosauskas MRG. Tecnologia da informação e comunicação na formação docente em saúde: relato de experiência. Revista Brasileira de Educação Médica 2011, 35 (1), 102-107.

\section{CONTRIBUIÇÃO DOS AUTORES}

Os autores Bráulio Brandão Rodrigues, Lanna Tarce Gonçalves de Morais, Paola Souza Manzi, Isabella Françoise Teles, Kálita Oliveira Lisboa, Nathalia Vitória Ramos, Rafael Felipe Gregório Machado participaram da concepção deste trabalho, desde a organização da atividade presente no relato até o desenho deste estudo e interpretação, e escrita do artigo.

O autor Denis Masashi Sugita participou da concepção deste trabalho, desde a organização da atividade presente no relato até o desenho deste estudo e interpretação, e escrita. Atuando como orientador do estudo.

\section{CONFLITO DE INTERESSES}

Todos os autores declaram a inexistência de conflitos de interesse.

\section{ENDEREÇO PARA CORRESPONDÊNCIA}

Bráulio Brandão Rodrigues - brandaobbr@gmail.com 